\title{
Sealed endoscopic full-thickness resection for gastric cancer: a pilot study in an ex vivo and in vivo porcine model
}

\section{(2) $\circledast \Theta$}

\author{
Authors \\ Kobayashi ${ }^{1}$, Junji Kamai ${ }^{1}$, Takeo Kosaka \\ Institutions \\ 1 Department of Gastroenterological Endoscopy, \\ Kanazawa Medical University, Ishikawa, Japan \\ 2 Department of Surgical Oncology, Kanazawa Medical \\ University, Ishikawa, Japan
}

Hidekazu Kitakata', Tohru Itoh ${ }^{1}$, Shinichi Kinami ${ }^{2}$, Ken Kawaura', Kazu Hamada', Sadafumi Azukisawa', Rika

submitted 30.5.2018

accepted after revision 24.9.2018

\author{
Bibliography \\ DOI https://doi.org/10.1055/a-0777-1954 | \\ Endoscopy International Open 2019; 07: E36-E42 \\ (c) Georg Thieme Verlag KG Stuttgart · New York \\ ISSN 2364-3722
}

Corresponding author

Hidekazu Kitakata, Department of Gastroenterological Endoscopy, Kanazawa Medical University, 1-1 Daigaku, Uchinada, Kahoku District, Ishikawa 920-0293, Japan Fax: +81-76-218-8416

kitakata@kanazawa-med.ac.jp

\section{ABSTRACT}

Background and study aims Endoscopic full-thickness resection (EFTR) is a useful procedure that allows minimal resection of the gastric wall because the tumor can be lo- cated endoscopically. However, the procedure carries a risk of peritoneal infection or dissemination. Thus, we devised a new EFTR technique in which the serosa is sealed using a silicone sheet to prevent the escape of gastric juice. Materials and methods Three whole stomachs were harvested from pigs for an ex vivo experiment, and seven pigs were used for an in vivo experiment. In both experiments, silicone sheets and gauze were pasted to the serosa using a fibrinogen-thrombin solution. A seromuscular incision was then made endoscopically using a HookKnife. We then evaluated whether stomach collapse could be prevented using this technique. Furthermore, the method was compared with conventional laparoscopic-assisted EFTR (LAEFTR) in terms of resection time and quality of endoscopic view.

Results In the ex vivo experiment, stomach collapse was suppressed and the seromuscular layer could be incised layer by layer. In the in vivo experiment, the time required for seromuscular incision with the new EFTR technique was significantly shorter than that with the conventional method. All layers of the stomach were smoothly resected under good endoscopic view.

Conclusions Sealed EFTR is a potentially useful technique for the minimally invasive resection of gastric tumor. All layers of the stomach could be incised while confirming the incision line from the inside of the stomach and avoiding exposure of the tumor to the abdominal cavity.

\section{Introduction}

Endoscopic full-thickness resection (EFTR) [1,2] and laparoscopic and endoscopic cooperative surgery (LECS) [3] are used to avoid excessive resection of the gastric wall and subsequent postoperative complications, such as stenosis or deformity. In these procedures, the tumor can be confirmed endoscopically to determine the appropriate resection line. However, the techniques also have some disadvantages, namely loss of endoscopic view caused by stomach collapse, and peritoneal infection or tumor dissemination due to outflow of gastric juice.

A combination of laparoscopic and endoscopic approaches to neoplasia with non-exposure technique (CLEAN-NET) [4] and non-exposed endoscopic wall-inversion surgery (NEWS) [5] have recently been devised as a procedure for local resection that does not expose the intragastric space. These procedures are characterized by a circumferential seromuscular incision made from outside the stomach under the guidance of either submucosal dye injection or intragastric navigation; that is, use of the endoscope to push against the gastric wall. We considered that it is important to resect the tumor from inside of the stomach while confirming the location of the tumor endoscopically.

Thus, we have devised a new technique, called sealed EFTR, whereby the serosa of the stomach is sealed using a silicone sheet to prevent stomach collapse and outflow of gastric juice. 


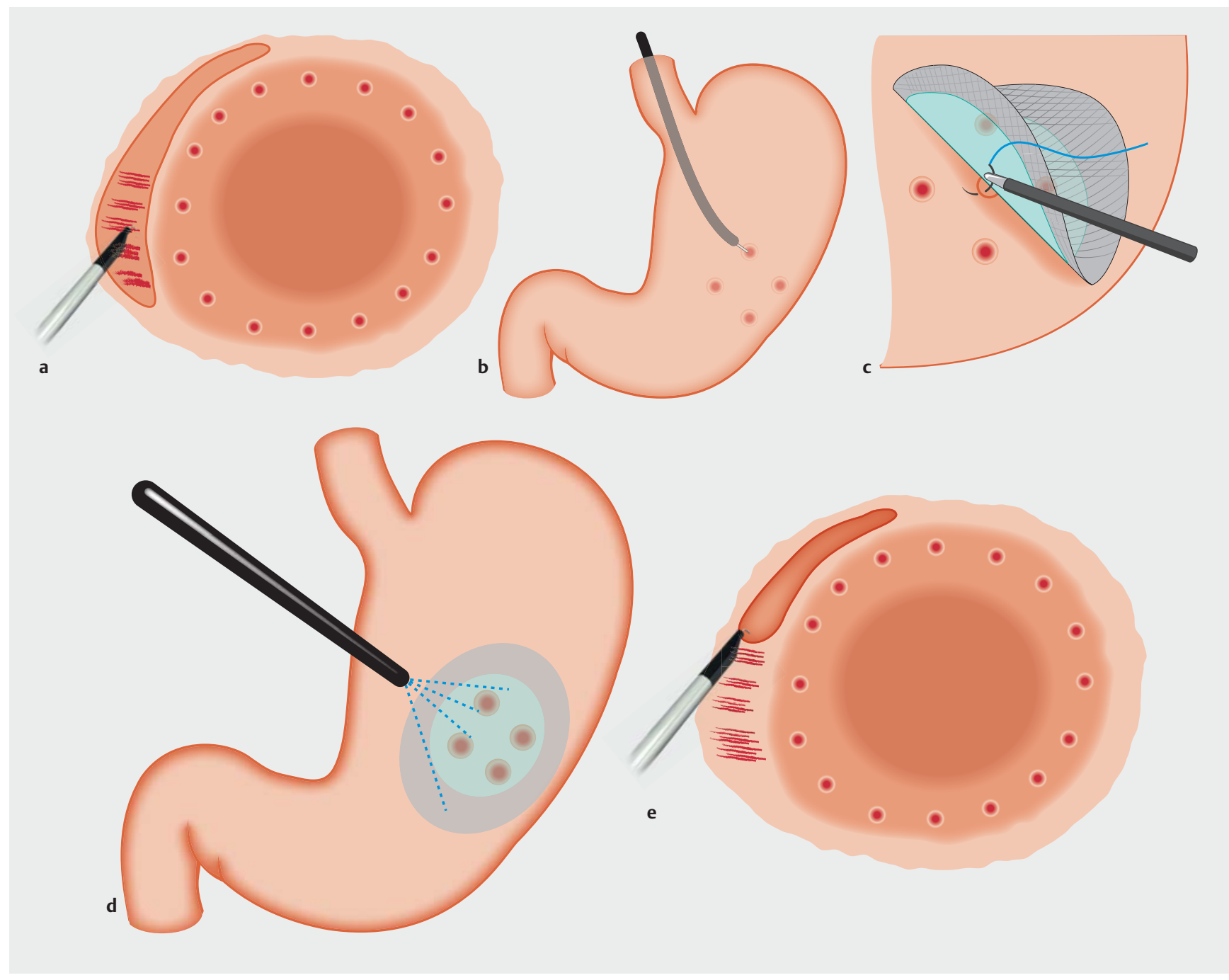

- Fig. 1 Procedure of sealed EFTR. a Circumferential mucosal incision - performed deeply and evenly to achieve exposure of muscular layer. b Marks on serosa placed on incision line using HookKnife. c Ligation of silicone and polyglycolic acid (PGA) sheets to the serosa of lesion, with the center of silicone sheet at the center of the serosa marks. $\mathbf{d}$ Pasting of silicone and PGA sheets to the serosa using fibrinogen-thrombin solution. e Dissection of seromuscular layer; simultaneous confirmation of mucosal marks.

\section{Materials and methods}

In an ex vivo experiment, three whole stomachs harvested from slaughtered pigs were used. After washing with water and 20,000 units of pronase, each stomach was set on an endoscopic submucosal dissection (ESD) training model.

First, markings were made around a virtual lesion using a HookKnife (KD-620; Olympus, Tokyo, Japan) in swift coagulation mode (effect 4;40W). The knife was powered using a VIO300D electrosurgical unit (ERBE; Tubingen, Germany). Next, $0.9 \%$ saline with indigo carmine was injected into the submucosa around the markings. A circumferential mucosalsubmucosal incision was then made using the HookKnife in dry cut mode (effect $4 ; 60 \mathrm{~W}$ ) in a manner similar to ESD ( $>$ Fig. 1a). Four marks were made on the incision line of the serosa from inside of the stomach using the HookKnife in coagulation mode ( $\mathbf{F i g}$. 1b). A silicone sheet $5 \mathrm{~cm}$ in diameter and several types of gauze 8 to $10 \mathrm{~cm}$ in diameter were placed on the serosa to cover the marks. These were then pasted using a fibrinogen thrombin solution ( $\boldsymbol{F}$ Fig.2a). Because a silicone sheet could not be directly adhered to the serosa, a gauze was overlapped and pasted. To cover the silicone sheet, one layer of gauze, four layers of gauze or non-woven gauze were used. Finally, a seromuscular incision was made using the HookKnife in dry cut mode ( $\mathbf{F i g} . \mathbf{1 e}$, $>$ Fig. $\mathbf{2 b}$, $>$ Fig. $\mathbf{2 c}$ ).

In this experiment, we examined whether it is possible to resect the virtual lesion from inside the stomach while keeping the lumen of the stomach expanded. In other words, we confirmed whether the silicone sheet could be adhered to the serosa with fibrinogen-thrombin solution; the sheet was not peeled off by endoscopic forceps manipulation or by expansion or contraction of the stomach; and whether the lumen of the stomach could be maintained in an expanded state after perforation.

After verifying the feasibility of our new technique in an ex vivo model, we conducted in vivo experiment. In the in vivo ex- 

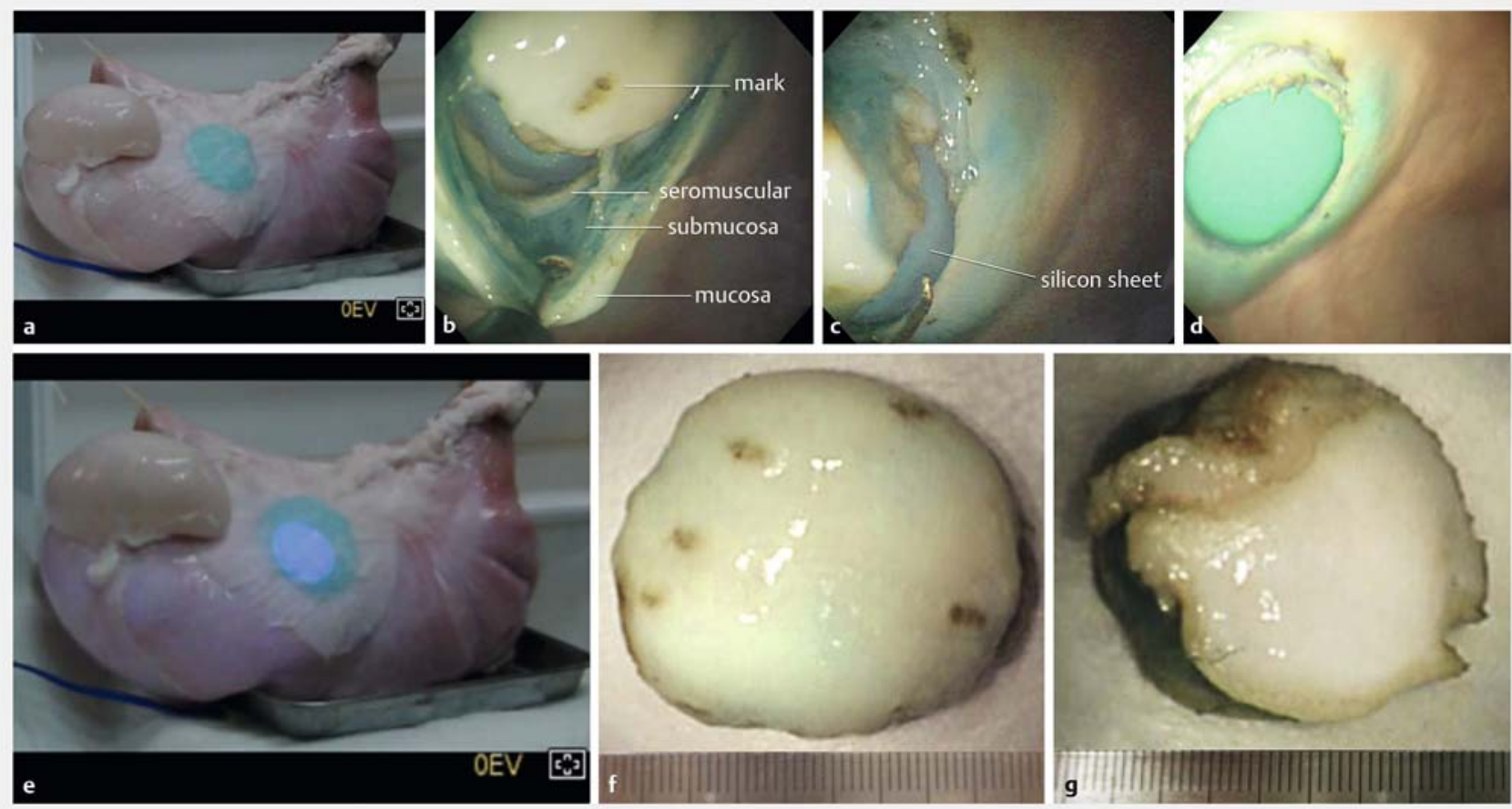

- Fig. 2 Sealed EFTR using non-woven gauze (ex vivo experiment). a Pasting of silicone sheet and gauze to the serosa using fibrinogen-thrombin solution. b, c Confirmation of each stomach layer and layer-by-layer incision. d, e Maintenance of the stomach in expanded state even after all-layer incision. f, $\mathbf{g}$ Resected specimen (f, mucosal side; $\mathbf{g}$, serosal side).

periment, polyglycolic acid (PGA) sheet (Neoveil; Gunze, Tokyo, Japan) was used instead of non-woven gauze.

In the in vivo experiment, four domestic pigs $(30-40 \mathrm{~kg})$ were used for the new sealed EFTR method and three were used for the conventional LA-EFTR method. All procedures were performed under general anesthesia and the animals were sacrificed in a painless manner.

Under general anesthesia, a laparoscope was inserted via an incision in the umbilical region to observe the abdominal cavity. Four trocars were placed in the left upper, left middle, right upper, and right middle abdomen, respectively. The stomach was cut free of the lesser and greater omenta using laparoscopic coagulation shears (SonoSurg; Olympus, Tokyo, Japan). Virtual lesions measuring 2 to $3 \mathrm{~cm}$ in diameter were created in various positions of the stomach.

In the same manner as the ex vivo experiments, markings were made around the virtual lesions; saline with a small amount of indigo carmine was then injected locally into the submucosal layer, and a circumferential mucosal incision was performed using the HookKnife ( $\bullet$ Fig. 1a, $>$ Fig. 3a). The incision was then deepened until the muscular layer was exposed. As far as possible, it was made to an even depth throughout the circumference. The marks were placed without perforation at four points on the incision line of the serosa from inside the stomach using the HookKnife in coagulation mode ( $\triangleright$ Fig. $\mathbf{1 b}$, - Fig. 3b).

Subsequently, a silicone sheet and PGA sheet were ligated to the serosa so that the center of the silicone sheet matched the center of the serosal marks ( $\triangleright$ Fig. 1c, $\triangleright$ Fig. 3c). The silicone and PGA sheets had been ligated beforehand to ensure their centers matched. Next, these sheets were pasted using a fibrinogen-thrombin solution ( $\triangleright$ Fig.1 d, $\triangleright$ Fig.3d). Specifically, the sheet was pasted to the serosa several minutes after the solution was sprayed so that it resembled a gel film.

Seromuscular dissection was then performed using a HookKnife and/or ITknife2 (KD-611L; Olympus, Tokyo, Japan). Specifically, a small hole was made on the incision line using the HookKnife, and the tip of the knife was inserted into the hole. The seromuscular layer was dissected using the HookKnife while confirming the presence of the mucosal marks around the lesion ( $\triangleright$ Fig. 1e, $\triangleright$ Fig. 3e).

After circumferential seromuscular dissection, the lesion was only attached by the thread with which the silicone sheet was ligated. The thread was cut using the HookKnife, and the resected specimen was removed through the oral cavity. The silicone and PGA sheets were peeled off the serosa using laparoscopic forceps and removed through the oral cavity. At that time, the stomach was kept elevated using laparoscopic forceps so that no gastric juice flowed into the abdominal cavity. Finally, the gastric wall defect was closed laparoscopically using hand-sewn sutures ( $\bullet$ Fig. $\mathbf{3 g}$ ).

In the experiment using the conventional method, after circumferential mucosal and submucosal incision, the serosa was incised under counter-traction provided by gripping the gastric wall with laparoscopic grasping forceps [6]. 

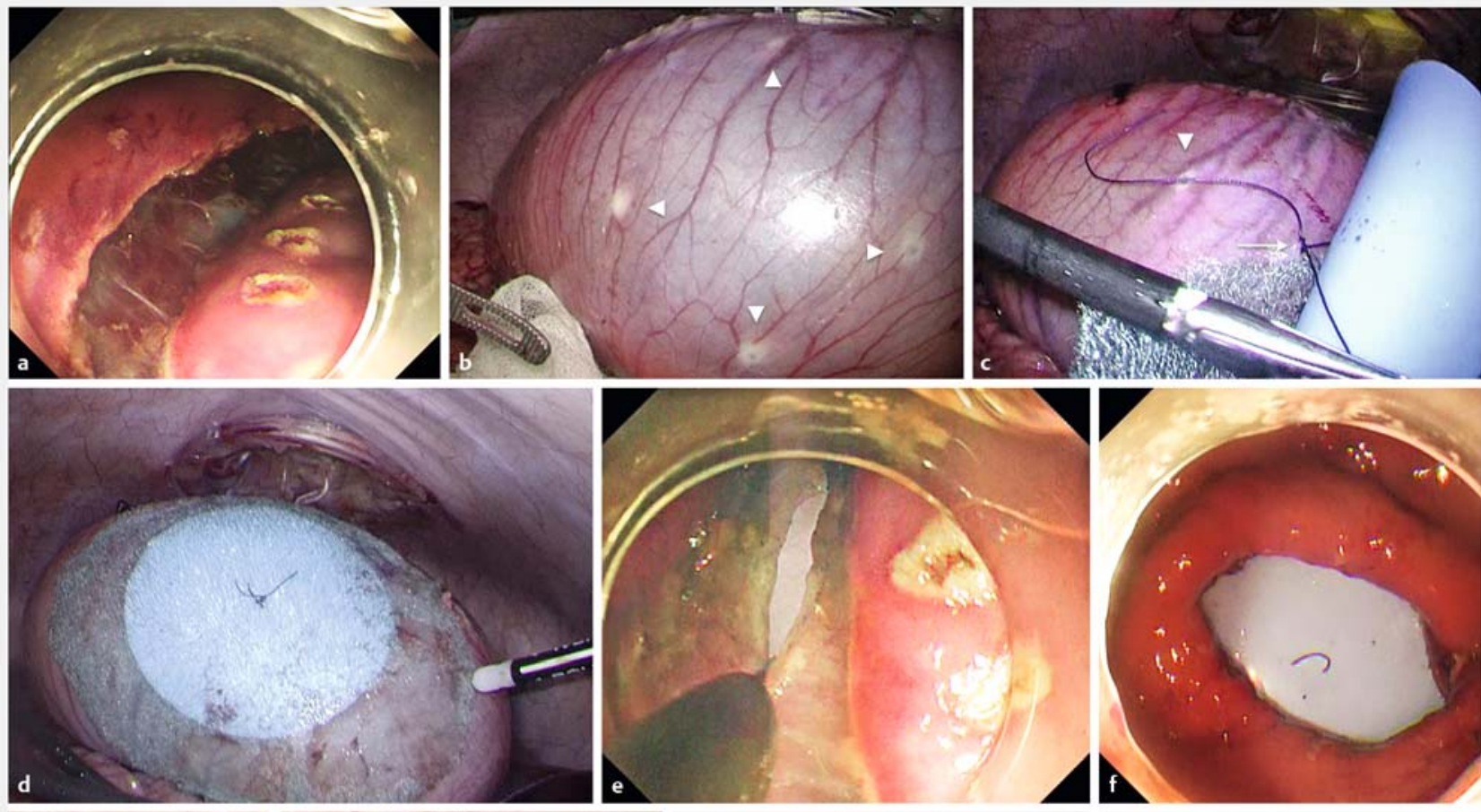

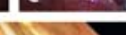
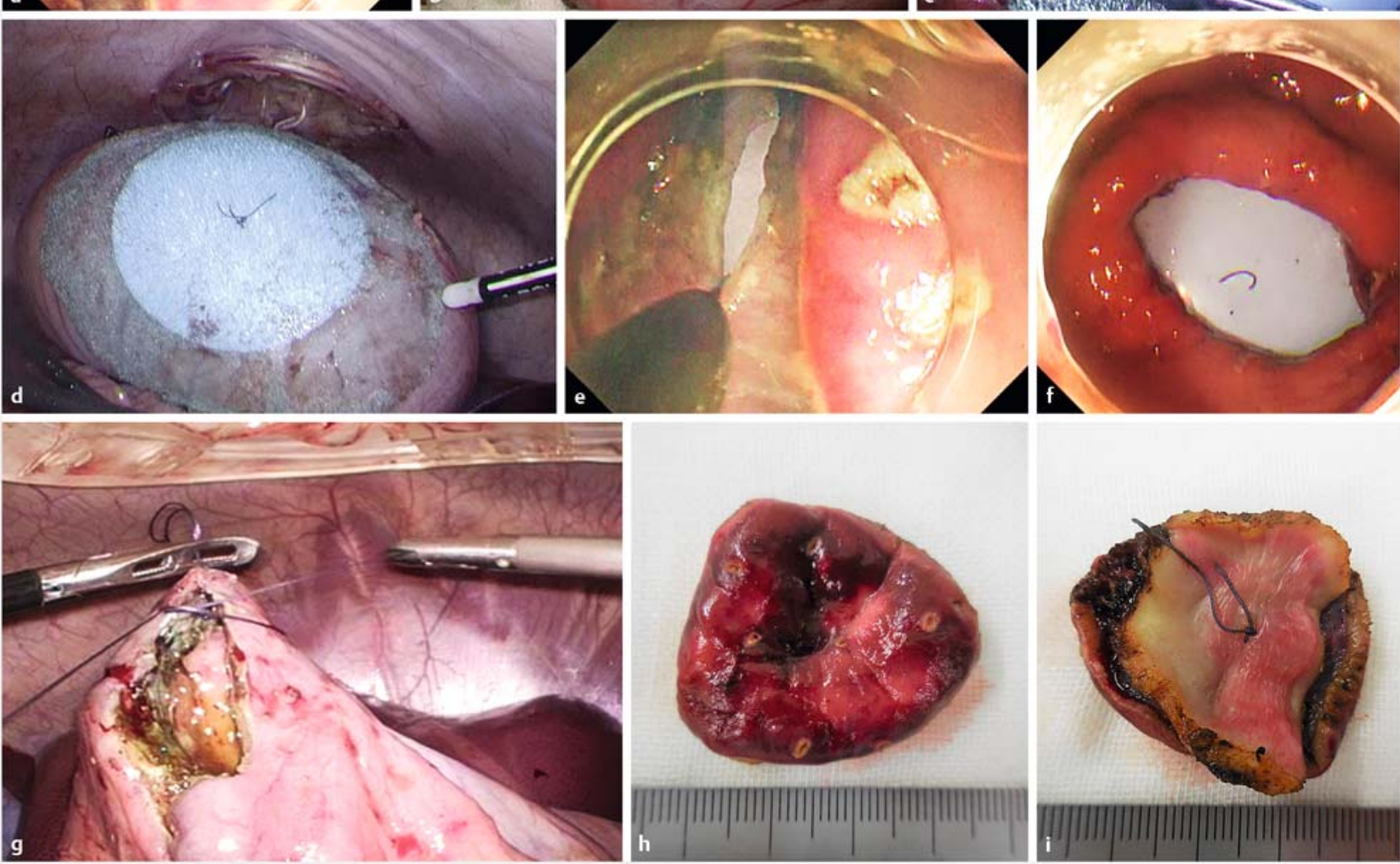

- Fig. 3 Sealed EFTR (in vivo experiment). a Circumferential mucosal incision-performed deeply and evenly to achieve exposure of muscular layer. $\mathbf{b}$ Marks on serosa (arrowhead) placed on incision line. $\mathbf{c}$ Ligation of silicone and polyglycolic acid (PGA) sheets to the serosa of lesion, with the center of silicone sheet at the center (arrow) of the serosa marks (arrow head). $\mathbf{d}$ Pasting of silicone and PGA sheets to the serosa using fibrinogen - thrombin solution. e Dissection of seromuscular layer; simultaneous confirmation of mucosal marks. $\mathrm{f}$ Maintenance of good endoscopic view after removal of resected specimen. $\mathbf{g}$ Laparoscopic closure of defect in the gastric wall. h, $\mathbf{i}$ Resected specimen (h, mucosal side; i, serosal side).

We compared resection time, stomach collapse, confirmation of the lesion and quality of endoscopic view between our new sealed EFTR technique and conventional LA-EFTR by using counter-traction with laparoscopic forceps or threads. Time for incision was measured separately from time for mucosal incision, time for sealing (from ligation the silicone sheet and PGA sheet to the serosa to adhesion them using fibrinogen-thrombin solution) and time for seromuscular incision. Stomach collapse and quality of endoscopic view were evaluated based on whether the lumen could be maintained in an expanded state and whether lesion, marks and incision line could always be confirmed. When the lumen of the stomach was kept open and the lesion could be confirmed only with a simple endo- scopic manipulation, confirmation of lesion is defined as "possible and easy" and quality of endoscopic view was defined as "good". Quality of the endoscopic view was defined as "not good" when the stomach collapsed and the lesion could not be confirmed only by endoscopic manipulation. In that case, if the lesion could be recognized by sufficient air supply or laparoscopic assistance, the confirmation of lesion was defined as "possible, but difficult," and if the lesion could not be recognized even by laparoscopic assistance, it was termed "impossible." Data were analyzed using $t$-test. Probability values less than 0.05 were considered statistically significant. 
- Table 1 Summary of sealed EFTR in vivo experiment.

\begin{tabular}{|c|c|c|c|c|c|c|c|}
\hline \multirow[t]{2}{*}{ Case } & \multirow[t]{2}{*}{ Position } & \multirow[t]{2}{*}{ Resection } & \multicolumn{3}{|c|}{ Time for incision (min) } & \multicolumn{2}{|c|}{ Size of resected specimen $(\mathrm{mm})$} \\
\hline & & & Mucosal incision & Sealing & Seromuscular incision & Mucosal side & Serosal side \\
\hline 1 & M ant. & Success & 12 & 13 & 12 & $29 \times 27$ & $25 \times 22$ \\
\hline 2 & M GC & Success & 15 & 19 & 6 & $32 \times 30$ & $27 \times 26$ \\
\hline 3 & M ant. & Success & 25 & 19 & 10 & $37 \times 34$ & $35 \times 32$ \\
\hline 4 & U ant. & Success & 20 & 30 & 16 & $36 \times 33$ & $35 \times 30$ \\
\hline 5 & M post. & Success & 16 & 32 & 6 & $34 \times 32$ & $32 \times 29$ \\
\hline 6 & UGC & Success & 22 & 29 & 15 & $34 \times 33$ & $34 \times 28$ \\
\hline 7 & Lant. & Success & 17 & 17 & 7 & $39 \times 37$ & $35 \times 35$ \\
\hline 8 & M LC & Failure & & & & & \\
\hline \multirow[t]{2}{*}{9} & U post. & Success & 33 & 35 & 12 & $37 \times 35$ & $38 \times 34$ \\
\hline & Average & & 20.0 & 24.3 & 10.5 & $34.5 \times 32.6$ & $32.6 \times 29.5$ \\
\hline
\end{tabular}

EFTR, endoscopic full-thickness resection; L, lower; M, middle; U, upper; GC, greater curve; LC, lesser curve; ant., anterior wall; post., posterior wall

The animal procedures were approved by the ethics committee of the Japan Consortium for Advances in Surgical Endoscopy.

\section{Results}

\section{Ex vivo experiment}

To seal the serosa, one layer of gauze, four layers of gauze, or non-woven gauze were used in duplicate. In all cases, the gauze was stuck to the serosa within approximately 5 minutes and was never peeled off by endoscopic forceps manipulation or by expansion or contraction of the stomach.

In cases that were sealed using one layer of gauze, incision in the serosa caused air outflow and subsequent stomach collapse. The stomach was expanded by the air supply, but it could not maintain the expanded state, and it was therefore difficult to incise the seromuscular layer. In cases that were sealed using four layers of gauze, air leakage was prevented more effectively and stomach collapse was suppressed. The lumen of the stomach was expanded by air supply with the endoscope, and the lesion could be confirmed. It was possible to incise the seromuscular layer while confirming the lesion. In cases that were sealed using non-woven gauze, the air outflow was prevented and the stomach did not collapse. It was possible to confirm the layers and incise the seromuscular layer more easily and rapidly, with a better endoscopic view ( $>$ Fig.2b, $\triangleright$ Fig.2c,

\section{> Fig. $2 \mathrm{~d}$, > Fig. 2e).}

The silicone sheet was not incised during seromuscular dissection with the HookKnife. Because there was a silicone sheet between the serosa and the gauze, it was possible to operate the HookKnife without breaking the gauze.

Furthermore, it was possible to resect specimens of almost identical sizes on the mucosal (mean specimen size: $40 \times 38$ $\mathrm{mm}$ ) and serosal sides (mean specimen size: $38 \times 37 \mathrm{~mm}$ )

( $\triangleright$ Fig. 2f, $\triangleright$ Fig. 2 g).

\section{In vivo examination}

Eight lesions, excluding that of the angle lesser curvature, were completely resected from inside the stomach using the ITknife2 and/or the HookKnife, without laparoscopic assistance. The silicone sheet and gauze could not be adhered to the angle lesser curvature, so tumor resection was therefore impossible. During the endoscopic procedure, the lumen of the stomach was kept open and a good endoscopic view was maintained ( $>$ Fig.3e, - Fig.3f). It was possible to dissect the seromuscular layer while confirming the mucosal marks around the lesion ( $\triangleright$ Fig. 3e). The sizes of the mucosa and serosa in the resected specimen were almost the same, measuring $35 \times 33 \mathrm{~mm}$ and $33 \times 30 \mathrm{~mm}$, respectively ( $\mathbf{F i g .} \mathbf{3} \mathbf{h}, \boldsymbol{\nabla} \mathbf{F i g} . \mathbf{3 i}$ ).

Mean time required for seromuscular incision with the sealed EFTR method was significantly shorter than that with the conventional method (10.5 minutes \pm 3.7 vs. 27.0 minutes $\pm 3.2, P<0.01)$ ( $\triangleright$ Table 1 and $\triangleright$ Table 2 ).

Using the conventional method, it took a mean of 27 minutes to incise the seromuscular layer because of poor endoscopic view and poor operability caused by interference of the endoscope and the laparoscopic forceps ( $\triangleright$ Table 2). Confirmation of the lesion was possible but difficult, because it was necessary to provide air supply and laparoscopic assistance. With the sealed EFTR method, it was possible to smoothly incise the seromuscular layer of the stomach under good endoscopic view, since air outflow was suppressed and there was no interference of the endoscope and the laparoscopic forceps.

When the lesions were in the upper region or on the posterior wall, it took more time to seal the serosa than when they were in the middle or lower regions or on the anterior wall ( Table 1). 
- Table 2 Comparison of serosa sealing method and the conventional method.

\begin{tabular}{|c|c|c|c|c|}
\hline & & Sealed EFTR & Conventional method & $P$ value \\
\hline & & $(n=8)$ & $(n=6)$ & \\
\hline \multicolumn{5}{|c|}{ Time for resection (min) } \\
\hline \multirow[t]{2}{*}{ - Total } & (Average) & $54.8 \pm 14.2$ & $42.0 \pm 3.7$ & 0.07 \\
\hline & (Range) & $(37-80)$ & $(38-48)$ & \\
\hline \multicolumn{2}{|c|}{ - Mucosal incision } & $20.0 \pm 6.2$ & $15.0 \pm 3.8$ & 0.11 \\
\hline & & $(12-33)$ & $(10-22)$ & \\
\hline \multirow[t]{2}{*}{ - Sealing } & & $24.3 \pm 7.6$ & - & \\
\hline & & $(13-35)$ & & \\
\hline \multirow{2}{*}{\multicolumn{2}{|c|}{ - Seromuscular incision }} & $10.5 \pm 3.7$ & $27.0 \pm 3.2$ & $<0.01$ \\
\hline & & $(6-15)$ & $(22-32)$ & \\
\hline \multicolumn{2}{|c|}{ Stomach collapse } & $(-)$ & $(+)$ & \\
\hline \multicolumn{2}{|c|}{ Quality of endoscopic view } & Good & Not good & \\
\hline \multicolumn{2}{|c|}{ Confirmation of the lesion } & Possible and easy & Possible, but difficult & \\
\hline
\end{tabular}

\section{Discussion}

EFTR was developed as a minimally invasive endoluminal surgery to treat gastrointestinal tumors (GISTs). It is a flexible endoscopic technique that can be used to excise all layers of the gastric wall while confirming the lesion from inside the stomach. Furthermore, it allows resection, with minimal surgical margin, of lesions that have penetrated into the deeper layers of the stomach wall $[1,2]$.

LECS was also developed as a minimally invasive surgery to treat gastric submucosal tumors [3]. In this method, resection of the tumor and closure of the defect are performed simultaneously using a laparoscopic linear stapler after endoscopic full-thickness incision covering approximately three-quarters of the defect circumference. Thus, LECS is thought to be more convenient and has gradually become accepted in Japan [3].

However, these methods create a hole in the gastric wall from which the stomach contents may flow into the abdominal space. For this reason, they may cause infection or tumor dissemination into the abdominal space $[4,5]$. Therefore, indications for these methods should be determined carefully in cases of GISTs with ulceration or epithelial tumors. Indeed, CLEAN-NET [4] and NEWS [5] were developed to avoid exposing the intragastric space in such cases. In these methods, a circumferential seromuscular incision is made from outside the stomach under the guidance of submucosal dye injection. Therefore, it is difficult to confirm the location of the tumor during incision. We considered that it is important to resect the tumor while confirming tumor location.

To avoid tumor dissemination and ensure resection of the full layer with an optimal surgical margin, we developed a new method of EFTR in which the serosa is sealed by a silicone sheet using a fibrinogen - thrombin solution. In the current study, we explored the feasibility of our new technique in an ex vivo and in vivo porcine model

Using this method, we suppressed outflow of air and gastric juices by adhering a silicone sheet to the serosa. In this way, we ensured a good endoscopic view while preventing intraperitoneal infection or dissemination.

During circumferential incision, the submucosal layer must be incised deeply and evenly to expose the muscular layer. Therefore, compared with the conventional method, it took more time to carry out circumferential incision of the mucosal and submucosal layer using our new method. It also took more time to seal the serosa. However, it was possible to incise the seromuscular layer while confirming the lesion with good endoscopic view in a shorter time than with the conventional method.

After resection of the tumor using our new technique, the gastric lumen is temporarily released to allow the sheet to be retrieved. Because the tumor has already been removed at this point, risk of dissemination is low. Nonetheless, gastric juice must be prevented from escaping; to that end, the stomach wall is pulled using laparoscopic forceps. In clinical cases, intraperitoneal lavage must be performed after closure of the stomach wall.

Ikehara et al. [7] reported that there was no peritoneal dissemination in 90 cases of endoscopic resection-related perforation and concluded that peritoneal dissemination was unlikely to occur after perforation during endoscopic resection. Although there are some reports of peritoneal dissemination after endoscopic resection-related perforation, it is presumed that they occurred due to inappropriate manipulation $[8,9]$. In the report of submucosa-infiltrating gastric cancer of perito- 
neal seeding after ESD perforation, it was reported that the tumor was adhered to the omentum because it was closed with an endoscopic clip while leaving the tumor after perforation and the operation was performed later [8]. In a report of peritoneal dissemination after laparoscopic partial resection of duodenal cancer, it was reported that the abdominal cavity came into contact with the resected specimen [9]. If the tumor is properly manipulated so as not to come into contact with the peritoneal cavity, peritoneal dissemination after perforation is unlikely to occur, as reported by lkehara et al [7].

Fibrinogen-thrombin solution, which consists of biological components, was used to avoid damage of the serosa, when the silicone sheet was adhered to the serosa. Silicone sheets cannot be directly adhered to the serosa, but they can be adhered using overlapping non-woven gauze. The silicone sheet and gauze were never peeled off by endoscopic forceps manipulation or by expansion or contraction of the stomach. However, they could be easily peeled from the serosa using laparoscopic forceps. Moreover, because the silicone sheet is not directly adhered to the serosa, the HookKnife can be moved around, allowing sliding during the serosal incision.

Submucosal gastric cancer negative for sentinel lymph nodes may be considered one indication for this method. Indeed, because submucosal gastric cancer is often associated with lymph node metastasis, gastrectomy with prophylactic regional lymphadenectomy is usually conducted, according to the guidelines of the Japanese Gastric Cancer Association [10]. However, reported incidence of lymph node metastasis in cases of submucosal gastric cancer is only $18 \%$ [11], and many patients with submucosal gastric cancer undergo a larger lymphadenectomy than is necessary. In the remaining approximately $80 \%$ of patients without lymph mode metastasis, lymphadenectomy can be avoided altogether [6].

In this regard, Kitagawa et al. [12] reported that the sentinel node concept was valid in cases of clinical T1N0 or T2N0 gastric cancer with a tumor diameter of $4 \mathrm{~cm}$ or less. In cases negative for sentinel lymph nodes, lymph node dissection can be reduced, and local resection of submucosal gastric cancer is possible. Furthermore, because the nerve damage caused by lymphadenectomy can be reduced, gastric function can be preserved and postoperative problems, such as early satiety and stasis of the stomach, can be reduced.

However, this adaptation of the procedure has some limitations. For instance, it is difficult to apply to lesions located in the angle lesser curvature, because the sheet cannot be adhered to the serosa. To overcome this problem, it is necessary to prepare a silicone sheet that fits the shape of the stomach; this aspect needs to be addressed in the future. It is possible to resect the lesions located in other positions where the silicone sheet can be adhered. In particular, it is a good indication for lesions located in the anterior wall or the great curvature. In cases of posterior wall lesions, the tumor can be resected; however, it takes a long time to adhere the sheet to the serosa because of stomach mobilization and space limitations in the abdominal cavity.

\section{Conclusion}

Sealed EFTR is a potentially useful technique that allows minimally invasive resection of gastric tumor, because it was possible to incise all layers of the stomach while confirming the incision line endoscopically and avoiding exposure of the tumor to the abdominal cavity.

\section{Acknowledgements}

This work was supposed by JSPS Grant-in-Aid for Scientific Research Grant Number JP16K09409.

\section{Competing interests}

None

References

[1] Ikeda K, Mosse CA, Park PO et al. Endoscopic full-thickness resection: circumferential cutting method. Gastrointest Endosc 2006; 64: 82 - 89

[2] Abe N, Mori T, Takeuchi H et al. Successful treatment of early stage gastric cancer by laparoscopy-assisted endoscopic full-thickness resection with lymphadenectomy. Gastrointest Endosc 2008; 68: $1220-1224$

[3] Hiki N, Yamamoto Y, Fukunaga T et al. Laparoscopic and endoscopic cooperative surgery for gastrointestinal stromal tumor dissection. Surg Endosc 2007; 22: 1729-1735

[4] Inoue $\mathrm{H}$, Ikeda $\mathrm{H}$, Hosoya T et al. Endoscopic mucosal resection, endoscopic submucosal dissection, and beyond: full-layer resection for gastric cancer with nonexposure technique (CLEAN-NET). Surg Oncol Clin N Am 2012; 21: 129-140

[5] Goto O, Mitsui T, Fujishiro M et al. New method of endoscopic fullthickness resection: a pilot study of non-exposed endoscopic wall-inversion surgery in an ex vivo porcine model. Gastric Cancer 2011; 14 : $183-187$

[6] Itoh T, Kitakata H, Kinami S et al. New treatment strategy for submucosa-infiltrating gastric cancer without sentinel node metastasis: a pilot study of laparoscopy-assisted endoscopic full-thickness resection in an in vivo porcine model. Endosc Forum Digest Dis 2015; 31: $17-26$

[7] Ikehara H, Gotoda T, Ono H et al. Gastric perforation during endoscopic resection for gastric carcinoma and the risk of peritoneal dissemination. Br J Surg 2007; 94: 992 - 995

[8] Morita A, Yabushita K, Kimura J et al. A case of stage IB gastric cancer caused peritoneal dissemination in 1 year after perforation during endoscopic submucosal dissection. Jpn J Gastroenterol Surg 2007; 40: $399-404$

[9] Satake M, Takata M, Komatsu M et al. A case of duodenal mucosal cancer caused peritoneal dissemination after laparoscopic local resection of duodenum. J Jpn Soc Endsco Surg 2013; 18: 663 (Japanese)

[10] Japanese Gastric Cancer Association. Japanese gastric cancer treatment guidelines 2014 (ver. 4). Gastric Cancer 2017; 20: 1- 19

[11] Ito $\mathrm{H}$, Inoue $\mathrm{H}$, Ikeda $\mathrm{H}$ et al. Clinicopathological characteristics and treatment strategies in early gastric cancer: a retrospective cohort study. J Exp Clin Cancer Res 2011; 30: 117-123

[12] Kitagawa Y, Takeuchi H, Takagi Y et al. Sentinel node mapping for gastric cancer: a prospective multicenter trial in Japan. J Clin Oncol 2013; $31: 3704-3710$ 